\title{
Toxicity of Vrishabhavathy river water and sediment to the growth of phaseolus vulgharis (french beans)
}

\author{
${ }^{1 *}$ AHIPATHY, M V; ${ }^{2}$ PUTTAIAH, E T \\ ${ }^{1}$ PES College, Hanumanthanagar, Bangalore-560050 INDIA. E-mail: ahipathy@yahoo.co.in Ph No.00-91-80-26620726 Mobile: \\ $+919448955203$ \\ ${ }^{2}$ P.G. Department of Environmental Science, Kuvempu University, Shankaraghatta, Karnataka State INDIA
}

\begin{abstract}
Vrishabhavathy river is a tertiary tributary of river Cauvery, passing through many thickly populated residential and industrial areas in Bangalore city, India. This river has taken the wrath of industrial and domestic effluents over half a century and hence has been considerably polluted. The present paper is aimed at determining the suitability of the river water for agricultural purposes by finding out the effect of the use of river water and sediment on the growth of French beans compared to a control with loamy soil and bore-well water free from heavy metals and organic matter. No heavy metal was present in the edible part of the plant. It was observed that the height of plants, number of leaves, number of flowers, number of pods and biomass were all more than that with control for all combinations with river water and sediment. The results have been statistically analysed and presented in the paper. All the parameters studied were highly influenced by either river water with loamy soil or reduced composition of sediment soil with river water over river water with sediment or the control. It is concluded that this river water and sediment might be used as a source of fertilizer for better growth of plants. Presence of heavy metals though high in water and sediment does not enter the food chain and hence poses no threat. @JASEM
\end{abstract}

Man beyond all imaginations due to industrialization, urbanization, increased population and deforestation has polluted water. It is the duty of each and every human being to prevent or atleast reduce pollution to the minimum by adopting suitable techniques to purify the already polluted stretches. This is necessitated by the fact that many rivers and even underground water reserves are found to be unfit even for agricultural purposes let alone human consumption. A step in this direction is to assess the degree of pollution in a given surface or underground water source in order to be able to predict the suitability of water for human consumption, agriculture, industrial or non-contact recreation purposes. Many sources of water though not suitable for human consumption, are fit for agriculture or industrial use. An attempt is being made to assess the suitability of River Vrishabhavathy, the river under investigation for agricultural purpose. In the present paper, the effect of pollutants with special reference to industrial and domestic effluents on the growth of French beans studied in the lab using pot model has been presented.

Several workers have studied the effect of pollutants such as heavy metals, sewage and industrial effluents on the growth of many plants. Aggarwal et al (2000) have investigated the effect of domestic waters on Physico-chemical characteristics of soil at Varuna River corridor along with growth of characteristic plants. Biney Charles (1991) has elaborated a method for characterizing natural and waste waters using plant germination technique in which fraction of the percentage increase in weight of seeds in test waters to that in control has been chosen as the criteria. Kannabiran et al (1998) studied the effect of domestic waste on the growth and yield of Vigna Mungo (L) Hepper. Nagaraja and Krishnamurthy (1988) have carried out similar work with fodder grass (NB-21). Srivastava et al (1993) have investigated the effect of sewage on Lettuce plant. Lewander et al (1996) carried out experiments in pots and sealed jars using Potamogeton Pectinatus and Myriophyllum Verticillatum with the aim of finding out the possibility of existence of a gradient of metal pollution. Karmegam and Daniel (2000) have analysed the mannurial characteristics of biodigested slurry and vermicompost on the growth and yield of Cowpea Vigna Unguiculata (L.) Walp variety $\mathrm{Cl}$. Diamond et al (1994) have used an integrated monitoring approach to determine site specific effluent metal limits with three forms of monitoring Chemical specific analysis of effluent samples, whole effluent chronic toxicity tests and stream bio assessment.

\section{MATERIALS AND METHODS}

Vrishabhavathy river, a tertiary tributary of river Cauvery originates near a hillock in Peenya industrial area, Bangalore city. This river with a stretch of over $50 \mathrm{kms}$ was a source of drinking water to the populace in Bangalore. It travels through industrial belts and thickly populated residential areas carrying the generated effluents with it. Ahipathy and Puttaiah (2006) have studied the degree of pollution of the river water. The present study was aimed at

*Corresponding author: E-mail: ahipathy@yahoo.co.in Ph No.00-91-80-26620726 Mobile: +919448955203 
determining the effect of the use of the river water and sediment on the growth of French beans. Depending on the accessibility and introduction of effluents, four sites were chosen for collection of water and sediment samples. Topography of the river with sampling sites is indicated in figure 1 .

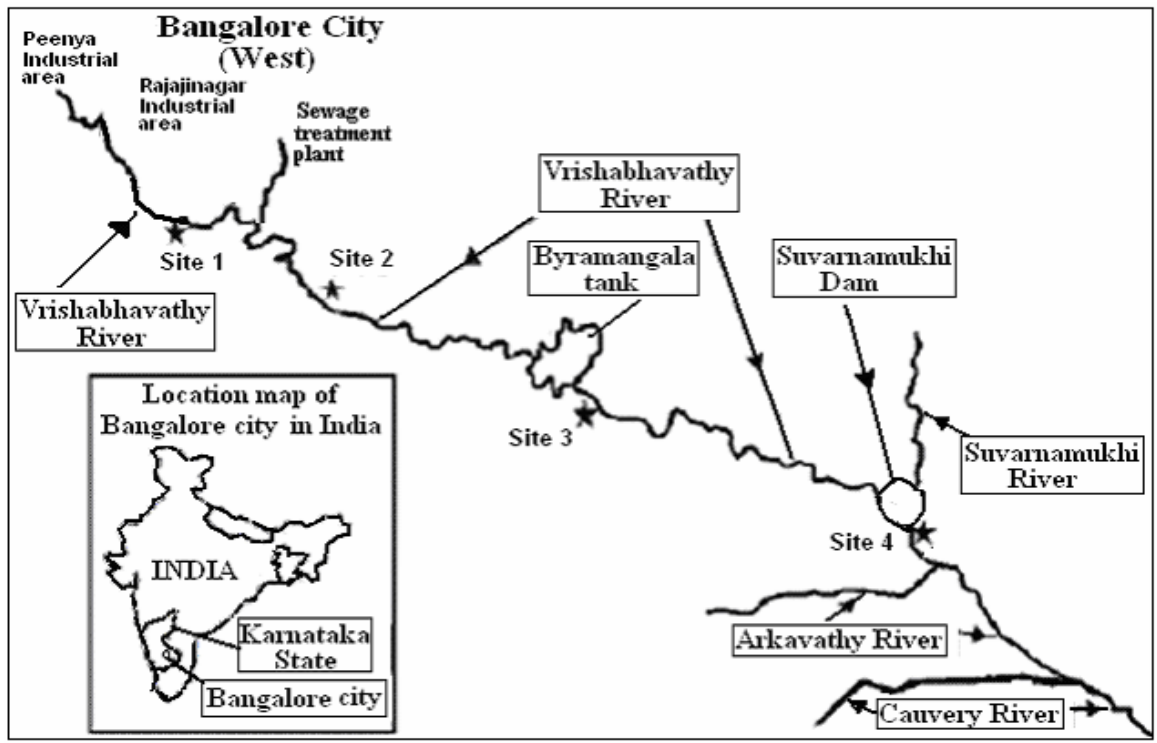

Fig 1: Topography of River Vrishabhavathy with sampling sites

From each site, about $10 \mathrm{kgs}$ of sediment soil samples were scooped out from the surface and transferred to plastic containers and surface water samples were collected in 10 litre plastic containers. Water samples were collected on alternate days whereas the sediment samples were collected only at the beginning of the experiment. French beans in packets of 100 were procured from Lalbagh nursery in Bangalore city. About 500 large sized healthy seeds were separated. As a measure of the quality of seeds with respect to germination and growth, the germination value was first determined. Higher the germination value, better the quality of seeds and more suited for the present toxicity test. The number of sprouted seeds per 100 seeds started with gives the germination value.

To determine the germination value of the seeds, the seeds were soaked in demineralised bore-well water containing no organic matter for $24 \mathrm{hrs}$. The seeds were then kept wet for about $72 \mathrm{hrs}$. The number of sprouted seeds was then counted and then the germination value was calculated. The germination value with the French beans used was found to be $90 \%$. De-mineralized Bore-well water containing no heavy metals and organic matter was used for dilution of the river water. Three dilutions of $75 \%$, $50 \%$ and $25 \%$ were prepared for each sampling station by mixing the river water with the bore-well water. In addition to this, $100 \%$ river water sample and $100 \%$ bore-well water were also used during the experiment. Good loamy soil not exposed to the polluted water was chosen for preparing different compositions of the sediments from each site. Sediments with $75 \%, 50 \%$ and $25 \%$ compositions were prepared. In addition to this, $100 \%$ river sediment and $100 \%$ good loamy soil were also used. Sediment samples with the composition of $75 \%, 50 \%$ and $25 \%$ were placed in different plastic covers along with two covers with $100 \%$ river sediment and four covers with $100 \%$ good soil totalling up to nine covers per station.

A common control used for all the stations together was $100 \%$ good loamy soil watered with $100 \%$ bore well water. The water samples, different compositions of sediment samples and the good soil were analysed for the various physico-chemical parameters such as $\mathrm{pH}, \mathrm{BOD}, \mathrm{COD}$, total solids, total hardness, total available nitrogen, available Phosphorus, potassium, conductance and heavy metals. Three seeds were sown in each of the 37 pots ( 9 pots for each of the 4 stations + common control) and the date and time of sowing the seeds was noted sown. The pots were covered at the top with plastic sheet (instead of the usually used glass house) to avoid rainwater from diluting the contents in the covers. 
Table 1. Composition of sediment and water used in Phytotoxicity

\begin{tabular}{lccccc}
\hline \multicolumn{1}{c}{ Pot No } & Treatment No & Sediment soil & Good soil & River water & Bore-well water \\
$1,2,3,4$ & X1 & $0 \%$ & $100 \%$ & $100 \%$ & $0 \%$ \\
$5,6,7,8$ & X2 & $100 \%$ & $0 \%$ & $100 \%$ & $0 \%$ \\
$9,10,11,129,10,11,12$ & X3 & $75 \%$ & $25 \%$ & $100 \%$ & $0 \%$ \\
$13,14,15,16$ & X4 & $50 \%$ & $50 \%$ & $100 \%$ & $0 \%$ \\
$17,18,19,20$ & X5 & $25 \%$ & $75 \%$ & $100 \%$ & $0 \%$ \\
$21,22,23,24$ & X6 & $0 \%$ & $100 \%$ & $75 \%$ & $25 \%$ \\
$25,26,27,28$ & X7 & $0 \%$ & $100 \%$ & $50 \%$ & $50 \%$ \\
$29,30,31,32$ & X8 & $0 \%$ & $100 \%$ & $25 \%$ & $75 \%$ \\
$34,35,36,37$ & X9 & $100 \%$ & $0 \%$ & $0 \%$ & $100 \%$ \\
\multicolumn{1}{c}{33} & Control & $0 \%$ & $100 \%$ & $0 \%$ & $100 \%$ \\
\hline
\end{tabular}

At the end of 14, 24, 36 and 45 days, height of the plant, number of leaves, number of branches, number of flowers and number of pods along with the area of the leaves and length of the pods were noted down. After six weeks, when the plants started withering, edible and non-edible parts of plants from each pot were separately collected, air-dried, powdered and weighed and added to get total biomass. A known amount of this dry sample was extracted with conc. $\mathrm{H}_{2} \mathrm{SO}_{4}$ and hydrogen peroxide and analyzed for $\mathrm{N}, \mathrm{P}$, and $\mathrm{K}$. The edible and non-edible parts were analysed for $\mathrm{Al}, \mathrm{Cd}, \mathrm{Cr}, \mathrm{Cu}$. Fe, $\mathrm{Mn}, \mathrm{Pb}$ and $\mathrm{Zn}$. N, K and $\mathrm{P}$ of edible and non-edible parts were added together to arrive at the total accumulation of $\mathrm{N}, \mathrm{K}$ and $\mathrm{P}$. The experimental findings of pot experiments were statistically analysed along with missing data analysis for proper explanation of data.

\section{RESULTS}

The results of the pot experiment conducted to elucidate the effect of the Vrishabhavathy River water and sediments on the growth characteristics of French beans are presented below: Plant height: The data on plant height as influenced by water and sediment compositions at different stages of growth are presented in table 2 .

Table 2: Influence of different compositions of river water and sediment from different sites on height of plants of French beans at various growth stages

\begin{tabular}{|c|c|c|c|c|c|c|c|c|c|c|c|}
\hline \multicolumn{12}{|c|}{ Height of plants } \\
\hline \multicolumn{12}{|c|}{ 14DAS } \\
\hline Treatments & $\mathrm{X} 1$ & $\mathrm{X} 2$ & $\mathrm{X} 3$ & $\mathrm{X} 4$ & $\mathrm{X} 5$ & X6 & $\mathrm{X} 7$ & $\mathrm{X} 8$ & X9 & Control & Mean \\
\hline Site 1 & 29.8 & 13.12 & 22.01 & 26.67 & 13.33 & 16.47 & 20.32 & 19.89 & 13.97 & 18.7 & 19.43 \\
\hline Site 2 & 27.92 & 22.01 & 19.47 & 19.05 & 0 & 15.41 & 28.36 & 16.13 & 27.94 & 18.7 & 19.5 \\
\hline Site 3 & 26.68 & 12.7 & 18.2 & 24.55 & 25.4 & 14.6 & 25.7 & 27.77 & 0 & 18.7 & 19.43 \\
\hline Site 4 & 26.68 & 12.7 & 15.24 & 25.4 & 23.95 & 19.47 & 19.89 & 20.74 & 12.96 & 18.7 & 19.57 \\
\hline Mean & 27.77 & 15.13 & 18.73 & 23.92 & 15.67 & 16.49 & 23.57 & 21.13 & 13.72 & 18.7 & 19.48 \\
\hline & Treatment & Sites & Interaction & & & & & & & & \\
\hline F-test & $* *$ & NS & $* *$ & & & & & & & & \\
\hline \multicolumn{12}{|c|}{ 24DAS } \\
\hline Treatments & $\mathrm{X} 1$ & $\mathrm{X} 2$ & $\mathrm{X} 3$ & $\mathrm{X} 4$ & $\mathrm{X} 5$ & $\mathrm{X} 6$ & $\mathrm{X} 7$ & $\mathrm{X} 8$ & X9 & Control & Mean \\
\hline Site 1 & 39.5 & 20.32 & 28.79 & 28.79 & 18.63 & 27.93 & 25.4 & 25.4 & 21.56 & 25.3 & 26.16 \\
\hline Site 2 & 38.1 & 30.48 & 22.01 & 26.67 & 0 & 23.04 & 30.9 & 24.57 & 30.48 & 25.3 & 25.16 \\
\hline Site 3 & 27.61 & 22.44 & 26.68 & 38.1 & 25.4 & 16.93 & 26.55 & 29.6 & 0 & 25.3 & 23.86 \\
\hline Site 4 & 29.26 & 16.66 & 21.09 & 25.4 & 22.8 & 19.47 & 21.17 & 22.86 & 16.49 & 25.3 & 22.05 \\
\hline Mean & 33.62 & 22.48 & 24.64 & 29.74 & 16.71 & 21.84 & 26 & 25.61 & 17.13 & 25.3 & 24.31 \\
\hline & Treatment & Sites & Interaction & & & & & & & & \\
\hline F-test & $* *$ & $* *$ & $* *$ & & & & & & & & \\
\hline \multicolumn{12}{|c|}{ 36DAS } \\
\hline Treatments & $\mathrm{X} 1$ & $\mathrm{X} 2$ & $\mathrm{X} 3$ & $\mathrm{X} 4$ & $\mathrm{X} 5$ & $\mathrm{X} 6$ & $\mathrm{X} 7$ & $\mathrm{X} 8$ & X9 & Control & Mean \\
\hline Site 1 & 71.12 & 29.04 & 43.18 & 39.79 & 30.48 & 44.67 & 33.02 & 36.41 & 28.81 & 30.28 & 38.68 \\
\hline
\end{tabular}




\begin{tabular}{|c|c|c|c|c|c|c|c|c|c|c|c|}
\hline Site 2 & 56.67 & 49.95 & 26.25 & 38.1 & 0 & 37.83 & 35.56 & 35.93 & 33.02 & 30.28 & 34.36 \\
\hline Site 3 & 35.93 & 38.95 & 40.63 & 36.92 & 38.1 & 30.48 & 35.57 & 37.93 & 0 & 30.28 & 32.48 \\
\hline Site 4 & 0 & 27.85 & 35.53 & 32.95 & 35.53 & 31.33 & 31.33 & 28.79 & 26.63 & 30.28 & 28.02 \\
\hline \multirow[t]{2}{*}{ Mean } & 40.93 & 36.45 & 36.4 & 36.94 & 26.03 & 36.08 & 33.87 & 34.77 & 22.12 & 30.28 & 33.38 \\
\hline & $\begin{array}{c}\text { Treatment } \\
* *\end{array}$ & $\begin{array}{l}\text { Sites } \\
* *\end{array}$ & $\begin{array}{c}\text { Interaction } \\
* *\end{array}$ & & & & & & & & \\
\hline \multicolumn{12}{|c|}{ 45DAS } \\
\hline Treatments & $\mathrm{X} 1$ & $\mathrm{X} 2$ & $\mathrm{X} 3$ & $\mathrm{X} 4$ & $\mathrm{X} 5$ & $\mathrm{X} 6$ & $\mathrm{X} 7$ & X8 & X9 & Control & Mean \\
\hline Site 1 & 72.81 & 29.04 & 53.29 & 44.7 & 30.48 & 52.89 & 62.53 & 43.18 & 29.95 & 33.51 & 45.23 \\
\hline Site 2 & 56.8 & 52.49 & 31.32 & 45.72 & 0 & 45.71 & 48.26 & 42 & 35.56 & 33.51 & 39.13 \\
\hline Site 3 & 40.66 & 47.41 & 45.71 & 55.88 & 45.72 & 38.1 & 52.49 & 37.76 & 0 & 33.51 & 39.72 \\
\hline Site 4 & 0 & 35.44 & 29 & 43.28 & 0 & 40.64 & 36.4 & 35.56 & 31.1 & 33.51 & 28.49 \\
\hline \multirow[t]{2}{*}{ Mean } & 42.56 & 41.09 & 39.83 & 47.39 & 19.05 & 44.33 & 49.92 & 39.62 & 24.15 & 33.51 & 38.15 \\
\hline & Treatment & Sites & Interaction & & & & & & & & \\
\hline F-test & $* *$ & $* *$ & $* *$ & & & & & & & & \\
\hline
\end{tabular}

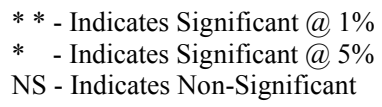

Table 2 F-test showed that treatments, Sites and interactions were significant at all stages of growth over control except in the initial stages wherein site variations were insignificant.

The statistical analysis revealed that the loamy soil treated with water from different sites of the river was found to be highly significant for plant height at all stages of growth. At initial stages of growth, treatments with different sites were at par with control but at later stages, site 4 treatments were significantly higher over the rest and control. Interactions between sites and treatments showed that loamy soil with water from site 4 , gave taller plants with plant heights of $29.8 \mathrm{cms}, 39.5 \mathrm{cms}, 71.12 \mathrm{cms}$ and $72.81 \mathrm{cms}$ at different stages of growth.

Among the various treatments studied with water and sediments, loamy soil with undiluted river water from different sites showed significant difference in height at all stages of growth and was statistically on par with loamy soil treated with 1:1 diluted river water and 1:1 sediment composition treated with river water, at later stages of growth.

Number of leaves: The data on number of leaves with different compositions of river water and sediments at different stages of growth are presented in tables 3 . Table 3 F-test indicated significantly higher values for treatments, sites and interactions over control at all stages except at 24DAS wherein sites did not show any significant difference from control. Loamy soil treated with river water from different sites gave significantly better number of leaves than the rest at all stages of growth. Treatments with different sites indicate that site 4 treatments were statistically significant over the rest and control at all stages except at 24 DAS when all are at par with control. Interactions show that loamy soil treated with water from site 4 surpassed the rest with values of $9.33,13$, 17.33 and 22 at the end of 14DAS, 24 DAS, 36DAS and 45DAS respectively.

Table 3: Influence of different compositions of river water and sediment from different sites on number of leaves of French beans at various growth stages

\begin{tabular}{|c|c|c|c|c|c|c|c|c|c|c|c|}
\hline \multicolumn{12}{|c|}{ Number of leaves } \\
\hline \multicolumn{12}{|c|}{ 14DAS } \\
\hline Treatments & $\mathrm{X} 1$ & $\mathrm{X} 2$ & $\mathrm{X} 3$ & $\mathrm{X} 4$ & $\mathrm{X} 5$ & X6 & $\mathrm{X} 7$ & $\mathrm{X} 8$ & X9 & Control & Mean \\
\hline Site 1 & 5.33 & 2 & 3 & 8 & 2 & 2 & 4 & 5 & 2 & 3 & 3.63 \\
\hline Site 2 & 5 & 8 & 2 & 4 & 0 & 2 & 5 & 2 & 5 & 3 & 3.6 \\
\hline Site 3 & 5 & 2 & 5 & 5 & 8 & 4 & 5 & 5 & 0 & 3 & 4.2 \\
\hline Site 4 & 5 & 3 & 3 & 8 & 5 & 8 & 4 & 4 & 5 & 3 & 4.8 \\
\hline Mean & 5.08 & 3.75 & 3.25 & 6.25 & 3.75 & 4 & 4.5 & 4 & 3 & 3 & 4.06 \\
\hline F-test & $\begin{array}{c}\text { Treatment } \\
\quad * *\end{array}$ & $\begin{array}{l}\text { Sites } \\
* *\end{array}$ & $\begin{array}{c}\text { Interaction } \\
* *\end{array}$ & & & & & & & & \\
\hline \multicolumn{12}{|c|}{ 24DAS } \\
\hline Treatments & $\mathrm{X} 1$ & $\mathrm{X} 2$ & $\mathrm{X} 3$ & $\mathrm{X} 4$ & $\mathrm{X} 5$ & X6 & $\mathrm{X} 7$ & $\mathrm{X} 8$ & X9 & Control & Mean \\
\hline Site 1 & 13 & 6 & 8 & 7 & 7 & 9 & 10 & 7 & 3.7 & 6 & 7.7 \\
\hline
\end{tabular}




\begin{tabular}{|c|c|c|c|c|c|c|c|c|c|c|c|}
\hline Site 2 & 11 & 9 & 7 & 10 & 0 & 7 & 10 & 8 & 7.3 & 6 & 7.5 \\
\hline Site 3 & 9 & 6 & 10 & 9 & 9 & 8 & 9.7 & 8.3 & 0 & 6 & 7.5 \\
\hline Site 4 & 8.3 & 8.3 & 8 & 6 & 5.7 & 7.3 & 7.7 & 5.7 & 6 & 6 & 6.9 \\
\hline Mean & 10.3 & 7.3 & 8.3 & 8 & 5.4 & 7.8 & 9.3 & 7.3 & 4.3 & 6 & 7.4 \\
\hline F-test & $\begin{array}{c}\text { Treatment } \\
\quad * *\end{array}$ & $\begin{array}{l}\text { Sites } \\
\text { NS }\end{array}$ & $\begin{array}{c}\text { Interaction } \\
\quad * *\end{array}$ & & & & & & & & \\
\hline \multicolumn{12}{|c|}{ 36DAS } \\
\hline Treatments & $\mathrm{X} 1$ & $\mathrm{X} 2$ & $\mathrm{X} 3$ & $\mathrm{X} 4$ & $\mathrm{X} 5$ & X6 & $\mathrm{X} 7$ & $\mathrm{X} 8$ & X9 & Control & Mean \\
\hline Site 1 & 17.33 & 6.33 & 11 & 9 & 12 & 12 & 16.3 & 11 & 6 & 10 & 11.1 \\
\hline Site 2 & 16 & 14 & 10 & 11 & 0 & 12 & 12 & 14 & 11 & 10 & 11.03 \\
\hline Site 3 & 14.33 & 11.33 & 12 & 12 & 14 & 9 & 13.6 & 15 & 0 & 10 & 11.13 \\
\hline Site 4 & 0 & 6 & 14 & 15 & 8 & 11 & 12 & 10 & 10 & 10 & 9.6 \\
\hline Mean & 11.92 & 9.42 & 11.75 & 11.7 & 8.5 & 11 & 13.5 & 13 & 6.8 & 10 & 10.72 \\
\hline & Treatment & Sites & Interaction & & & & & & & & \\
\hline F-test & $* *$ & $*$ & $* *$ & & & & & & & & \\
\hline \multicolumn{12}{|c|}{ 45DAS } \\
\hline Treatments & $\mathrm{X} 1$ & $\mathrm{X} 2$ & $\mathrm{X} 3$ & $\mathrm{X} 4$ & $\mathrm{X} 5$ & X6 & $\mathrm{X} 7$ & $\mathrm{X} 8$ & X9 & Control & Mean \\
\hline Site 1 & 22 & 9 & 11 & 9 & 10 & 13 & 17.3 & 14 & 7.3 & 10 & 12.27 \\
\hline Site 2 & 20 & 16.33 & 10 & 11 & 0 & 19 & 14 & 15 & 11 & 10 & 12.63 \\
\hline Site 3 & 15.67 & 14 & 12 & 15 & 15 & 18 & 14 & 14 & 0 & 10 & 12.73 \\
\hline Site 4 & 0 & 11 & 14.33 & 13 & 0 & 10 & 14 & 12 & 13 & 10 & 9.73 \\
\hline Mean & 14.42 & 12.58 & 11.83 & 12 & 6.25 & 15 & 14.8 & 14 & 7.8 & 10 & 11.84 \\
\hline F-test & $\begin{array}{l}\text { Treatment } \\
\quad * *\end{array}$ & $\begin{array}{l}\text { Sites } \\
* *\end{array}$ & $\begin{array}{c}\text { Interaction } \\
* *\end{array}$ & & & & & & & & \\
\hline
\end{tabular}

Leaf Area: The data on leaf area with different compositions of river water and sediments at different stages of growth are presented in tables 4 . Table 4 F-test showed significant difference between various treatments, sites and interactions and control at all stages of growth. Loamy soil treated with water from different sites scored over the rest, following the trends of plant height and number of leaves. Site 4 treatments excelled in giving larger leaf area over the other sites at earlier stages of growth, but at later stages, they were on par with site 2 and site 3 treatments. Interactions indicated very clearly that loamy soil treated with water from site 4 was better in giving larger leaf area at all stages of growth. The areas recorded are $323.66 \mathrm{~cm}^{2}, 385.5 \mathrm{~cm}^{2}, 464.5 \mathrm{~cm}^{2}$ and $541.45 \mathrm{~cm}^{2}$ respectively.

Table 4: Influence of different compositions of river water and sediment from different sites on leaf area of French beans at various growth stages

\begin{tabular}{|c|c|c|c|c|c|c|c|c|c|c|c|}
\hline \multicolumn{12}{|c|}{ Leaf area } \\
\hline Treatments & $\mathrm{X} 1$ & $\mathrm{X} 2$ & X3 & $\mathrm{X} 4$ & X5 & X6 & X7 & $\mathrm{X} 8$ & X9 & Control & Mean \\
\hline Site 1 & 323.67 & 43 & 58.1 & 69.35 & 29 & 64.5 & 59.66 & 85.2 & 38.7 & 37 & 80.82 \\
\hline Site 2 & 140 & 61.3 & 35.5 & 80.63 & 0 & 64.5 & 236.5 & 50.93 & 46.46 & 37 & 75.29 \\
\hline Site 3 & 50 & 51.6 & 69.26 & 50 & 75.8 & 59.13 & 69.35 & 49.16 & 0 & 37 & 51.13 \\
\hline Site 4 & 63.7 & 24.2 & 45.8 & 69.35 & 64.66 & 39.25 & 52.66 & 48.66 & 49.13 & 37 & 49.44 \\
\hline Mean & 144.34 & 45 & 52.17 & 67.33 & 42.37 & 56.85 & 104.6 & 58.49 & 33.57 & 37 & 64.17 \\
\hline F-test & $\begin{array}{c}\text { Treatment } \\
* *\end{array}$ & $\begin{array}{l}\text { Sites } \\
* *\end{array}$ & $\begin{array}{c}\text { Interaction } \\
* *\end{array}$ & & & & & & & & \\
\hline \multicolumn{12}{|c|}{ 24DAS } \\
\hline Treatments & $\mathrm{X} 1$ & $\mathrm{X} 2$ & $\mathrm{X} 3$ & $\mathrm{X} 4$ & $\mathrm{X} 5$ & $\mathrm{X} 6$ & $\mathrm{X} 7$ & $\mathrm{X} 8$ & X9 & Control & Mean \\
\hline Site 1 & 385.5 & 75 & 196.7 & 119.4 & 127.4 & 394.1 & 131.4 & 121 & 33.63 & 48.4 & 163.26 \\
\hline Site 2 & 144.5 & 170 & 114.53 & 132.2 & 0 & 202.5 & 111.3 & 239.5 & 68.27 & 48.4 & 123.11 \\
\hline Site 3 & 134.67 & 79.1 & 123.62 & 123.2 & 106.4 & 88.7 & 85.23 & 182.6 & 0 & 48.4 & 97.2 \\
\hline Site 4 & 0 & 71.9 & 158.75 & 68.93 & 52.97 & 47.4 & 116 & 65.3 & 48.53 & 48.4 & 67.82 \\
\hline Mean & 166.17 & 99 & 148.4 & 110.9 & 71.69 & 183.2 & 111 & 152.1 & 37.61 & 48.4 & 112.85 \\
\hline F-test & $\begin{array}{l}\text { Treatment } \\
\quad * *\end{array}$ & $\begin{array}{l}\text { Sites } \\
* *\end{array}$ & $\begin{array}{c}\text { Interaction } \\
\quad * *\end{array}$ & & & & & & & & \\
\hline \multicolumn{12}{|c|}{ 36DAS } \\
\hline Treatments & $\mathrm{X} 1$ & $\mathrm{X} 2$ & $\mathrm{X} 3$ & $\mathrm{X} 4$ & $\mathrm{X} 5$ & $\mathrm{X} 6$ & X7 & $\mathrm{X} 8$ & X9 & Control & Mean \\
\hline Site 1 & 464.5 & 111 & 234.7 & 406.5 & 208.1 & 265.1 & 386.8 & 261.3 & 72.6 & 152 & 256.3 \\
\hline
\end{tabular}




\begin{tabular}{cccccccccccc}
\hline Site 2 & 453 & 495 & 300 & 246.7 & 0 & 237.7 & 242.7 & 432.3 & 91.1 & 152 & 265.1 \\
Site 3 & 251.7 & 275 & 429.3 & 295.1 & 609.6 & 203.2 & 384.2 & 396.8 & 0 & 152 & 299.7 \\
Site 4 & 0 & 310 & 314.5 & 562.9 & 431 & 196.8 & 100.8 & 164.5 & 140.8 & 152 & 237.3 \\
Mean & 292.3 & 298 & 319.6 & 377.8 & 312.2 & 225.7 & 278.6 & 313.7 & 76.1 & 152 & 264.6 \\
& Treatment & Sites & Interaction & & & & & & & & \\
F-test & $* *$ & NS & $* *$ & & & & & & & & \\
& & & & & $45 \mathrm{DAS}$ & & & & & & \\
Treatments & $\mathrm{X} 1$ & $\mathrm{X} 2$ & $\mathrm{X} 3$ & $\mathrm{X} 4$ & $\mathrm{X} 5$ & $\mathrm{X} 6$ & $\mathrm{X} 7$ & $\mathrm{X} 8$ & $\mathrm{X} 9$ & Control & Mean \\
Site 1 & 541.4 & 132 & 188.8 & 115.9 & 152.3 & 461 & 405 & 264.5 & 29.7 & 75.9 & 236.6 \\
Site 2 & 447.5 & 416 & 141.9 & 382.2 & 0 & 469 & 238.7 & 404.9 & 158.1 & 75.9 & 273.4 \\
Site 3 & 406.1 & 361 & 455.3 & 653.2 & 423.4 & 157.2 & 451.3 & 335.8 & 0 & 75.9 & 331.9 \\
Site 4 & 0 & 321 & 551.1 & 332.2 & 0 & 206.5 & 226.6 & 145.1 & 338.7 & 75.9 & 219.7 \\
Mean & 348.7 & 308 & 334.3 & 370.9 & 143.9 & 323.4 & 330.4 & 287.6 & 131.6 & 75.9 & 265.4 \\
& Treatment & Sites & Interaction & & & & & & & & \\
F-test & $* *$ & $* *$ & $* *$ & & & & & & & & \\
\hline
\end{tabular}

Number of Flowers: The data on number of flowers with different compositions of river water and sediments at different stages of growth are presented in table 5. Table $5 \mathrm{~F}$-test indicated site variations to be non-significant at 36DAS, but significant for treatments and interactions at 45DAS. Statistical analysis again proved that loamy soil treated with water from different sites was significant over others and control at all stages of growth. At 36DAS, site 1 treatments showed better results with respect to number of flowers, but were on par with site 4 treatments. At 45DAS, site 3 treatments gave more number of flowers but were on par with treatments with sites and control. Interactions showed wide variations.

Table 5: Influence of different compositions of river water and sediment from different sites on number of flowers of French beans at various growth stages

\begin{tabular}{|c|c|c|c|c|c|c|c|c|c|c|c|}
\hline \multicolumn{12}{|c|}{ Number of flowers } \\
\hline \multicolumn{12}{|c|}{36 DAS } \\
\hline Treatments & $\mathrm{X} 1$ & $\mathrm{X} 2$ & $\mathrm{X} 3$ & $\mathrm{X} 4$ & $\mathrm{X} 5$ & $\mathrm{X} 6$ & $\mathrm{X} 7$ & $\mathrm{X} 8$ & X9 & Control & Mean \\
\hline Site 1 & 9.33 & 5 & 4.67 & 4.33 & 7.67 & 7.3 & 9.33 & 7.3 & 0 & 3.33 & 5.83 \\
\hline Site 2 & 6.33 & 8.67 & 6.33 & 6.33 & 0 & 8.7 & 4.67 & 0 & 5.3 & 3.33 & 4.97 \\
\hline Site 3 & 2 & 8.33 & 2 & 10.7 & 3.33 & 3 & 4.67 & 4.3 & 0 & 3.33 & 4.17 \\
\hline Site 4 & 0 & 4 & 9 & 10.3 & 0 & 10 & 6.33 & 6 & 7 & 3.33 & 5.6 \\
\hline \multirow[t]{2}{*}{ Mean } & 4.42 & 6.5 & 5.5 & 7.92 & 2.75 & 7.3 & 6.25 & 4.4 & 3.1 & 3.33 & 5.14 \\
\hline & Treatment & Sites & Interaction & & & & & & & & \\
\hline F-test & $* *$ & $*$ & $* *$ & & & & & & & & \\
\hline \multicolumn{12}{|c|}{ 45DAS } \\
\hline Treatments & $\mathrm{X} 1$ & $\mathrm{X} 2$ & $\mathrm{X} 3$ & $\mathrm{X} 4$ & $\mathrm{X} 5$ & $\mathrm{X} 6$ & $\mathrm{X} 7$ & $\mathrm{X} 8$ & X9 & Control & Mean \\
\hline Site 1 & 8.67 & 2 & 3.33 & 0 & 2.33 & 2 & 0 & 2 & 1.7 & 2.33 & 2.43 \\
\hline Site 2 & 6 & 2 & 2.67 & 2 & 0 & 2 & 2 & 7.7 & 3 & 2.33 & 2.97 \\
\hline Site 3 & 3 & 2 & 3 & 5 & 2 & 2.7 & 2 & 3 & 0 & 2.33 & 2.5 \\
\hline Site 4 & 0 & 4.33 & 0 & 2 & 0 & 2 & 2 & 4.7 & 6 & 2.33 & 2.33 \\
\hline \multirow[t]{2}{*}{ Mean } & 4.42 & 2.58 & 2.25 & 2.25 & 1.08 & 2.2 & 1.5 & 4.3 & 2.7 & 2.33 & 2.56 \\
\hline & Treatment & Sites & Interaction & & & & & & & & \\
\hline F-test & $*$ & NS & $*$ & & & & & & & & \\
\hline
\end{tabular}

Number of Pods: The data on number of pods with different compositions of river water and sediments at different stages of growth are presented in table 6. Table 6 F-test indicated site treatments and interactions to be significantly better than control at 36DAS while the treatments with different sites were non-significant at 45 DAS. Among treatments, loamy soil with water from different sites followed the trends of other parameters studied at both stages. Treatments with sites 4 and 2 were at par with site 3 at 36DAS but significant over site 1 and control. At 45DAS, site 1 treatments were significantly better than the rest of the sites and control but were statistically at par with other sites. Interactions between sites indicate that loamy soil treated with river water from site 4 far surpassed the rest. 
Table 6: Influence of different compositions of river water and sediment from different sites on number of pods of French beans at various growth stages

\begin{tabular}{|c|c|c|c|c|c|c|c|c|c|c|c|}
\hline \multicolumn{12}{|c|}{ Number of pods } \\
\hline \multicolumn{12}{|c|}{$36 \mathrm{DAS}$} \\
\hline Treatments & $\mathrm{X} 1$ & $\mathrm{X} 2$ & $\mathrm{X} 3$ & $\mathrm{X} 4$ & $\mathrm{X} 5$ & X6 & $\mathrm{X} 7$ & $\mathrm{X} 8$ & X9 & Control & Mean \\
\hline Site 1 & 6.33 & 0 & 2 & 3 & 0 & 1.7 & 0 & 1 & 0 & 0 & 1.4 \\
\hline Site 2 & 3 & 2 & 0 & 2 & 0 & 1 & 3 & 0 & 1 & 0 & 1.2 \\
\hline Site 3 & 1.33 & 0 & 2 & 3 & 2 & 0 & 0 & 4 & 0 & 0 & 1.23 \\
\hline Site 4 & 0 & 1 & 2 & 0 & 0 & 0 & 0 & 0 & 0 & 0 & 0.3 \\
\hline \multirow[t]{2}{*}{ Mean } & 2.67 & 0.75 & 1.5 & 2 & 0.5 & 0.7 & 0.75 & 1.3 & 0.3 & 0 & 1.03 \\
\hline & Treatment & Sites & Interaction & & & & & & & & \\
\hline F-test & $* *$ & $* *$ & $* *$ & & & & & & & & \\
\hline \multicolumn{12}{|c|}{ 45DAS } \\
\hline Treatments & $\mathrm{X} 1$ & $\mathrm{X} 2$ & $\mathrm{X} 3$ & $\mathrm{X} 4$ & $\mathrm{X} 5$ & X6 & $\mathrm{X} 7$ & $\mathrm{X} 8$ & $\mathrm{X} 9$ & Control & Mean \\
\hline Site 1 & 8.67 & 2.67 & 3 & 3.67 & 3.67 & 4.3 & 4.67 & 2.3 & 2 & 2 & 3.7 \\
\hline Site 2 & 1.67 & 4.33 & 3 & 5 & 0 & 2.7 & 3.67 & 1 & 5.3 & 2 & 2.87 \\
\hline Site 3 & 2 & 6 & 2 & 4 & 5.67 & 2.3 & 3.33 & 3 & 0 & 2 & 3.03 \\
\hline Site 4 & 0 & 3 & 6 & 5 & 0 & 4 & 3 & 2.3 & 4.7 & 2 & 3 \\
\hline \multirow[t]{2}{*}{ Mean } & 3.08 & 4 & 3.5 & 4.42 & 2.33 & 3.3 & 3.67 & 2.2 & 3 & 2 & 3.15 \\
\hline & Treatment & Sites & Interaction & & & & & & & & \\
\hline F-test & $* *$ & NS & $* *$ & & & & & & & & \\
\hline
\end{tabular}

Dry Biomass: The data on dry biomass with different compositions of river water and sediments at different stages of growth are presented in tables 7 . Table 7 Treatments with loamy soil with water from different sites gave maximum biomass but were on par with loamy soil treated with $3: 1$ and 1:1 diluted river water samples from different sites. Treatments with different sites showed site 2 to give best biomass but was found to be on par with site 4 and site 3 treatments. Interactions showed loamy soil treated with water from site 4 to be significant over the rest and control, the maximum biomass yield being 9.81gms.

Table 7: Influence of different compositions of river water and sediment from different sites on Dry Biomass of French beans at harvest

\begin{tabular}{cccccccccccc}
\hline & & \multicolumn{1}{c}{ Dry Biomass } \\
Treatments & X1 & X2 & X3 & X4 & X5 & X6 & X7 & X8 & X9 & Control & Mean \\
Site 1 & 5.81 & 1.63 & 2.58 & 2.54 & 6.07 & 7.43 & 4.8 & 2.41 & 1.52 & 1.7 & 3.65 \\
Site 2 & 4.24 & 4.78 & 2.67 & 5.67 & 0 & 7.05 & 7.86 & 3.82 & 3.46 & 1.7 & 4.12 \\
Site 3 & 4.5 & 6.08 & 3.09 & 5.95 & 2.58 & 6.06 & 6.49 & 6.14 & 0 & 1.7 & 4.26 \\
Site 4 & 0.47 & 3.35 & 2.79 & 6.22 & 0.54 & 6.58 & 3.79 & 1.91 & 1.42 & 1.7 & 2.88 \\
Mean & 3.76 & 3.96 & 2.78 & 5.1 & 2.3 & 6.78 & 5.74 & 3.57 & 1.6 & 1.7 & 3.73 \\
\hline
\end{tabular}

Heavy metal: The average heavy metal content in water and sediment is indicated in table 8 . The heavy metal in the sediment is in the unavailable form as the $\mathrm{pH}$ is in the alkaline side $(\mathrm{pH}=7.1-7.4)$. This prevents the heavy metals present in the insoluble combined form from being absorbed. Heavy metal content was checked only in the edible part (pods) of the plant at the end of the growth period. It was found that the heavy metal content in pods was below detectable limits. 
Table 8: Average heavy metal content in water and sediment of Vrishabhavathy River

\begin{tabular}{ccccccccc}
\hline Site & $\mathrm{Al}$ & $\mathrm{Cd}$ & $\mathrm{Cr}$ & $\mathrm{Cu}$ & $\mathrm{Fe}$ & $\mathrm{Mn}$ & $\mathrm{Pb}$ & $\mathrm{Zn}$ \\
1 & 8945 & 0.60 & 323.0 & 33.2 & 13445 & 1015 & 22.30 & 0.40 \\
2 & 3627 & 0.43 & 73.0 & 24.0 & 5767 & 448.6 & 21.60 & 42.70 \\
3 & 4106 & 2.00 & 27.0 & 519.0 & 14438 & 629.4 & 70.20 & 182.9 \\
4 & 7673 & 12.00 & 141.5 & 429.0 & 5685 & 563.9 & 83.60 & 222.4 \\
& & & & Water & & & & \\
Site & $\mathrm{Al}$ & $\mathrm{Cd}$ & $\mathrm{Cr}$ & $\mathrm{Cu}$ & $\mathrm{Fe}$ & $\mathrm{Mn}$ & $\mathrm{Pb}$ & $\mathrm{Zn}$ \\
1 & 0.18 & 0.01 & 0.03 & 0.02 & 0.16 & 0.32 & 0.07 & 0.03 \\
2 & 0.24 & 0.01 & 0.03 & 0.04 & 0.52 & 0.33 & 0.07 & 0.09 \\
3 & 0.44 & 0.01 & 0.03 & 0.05 & 0.41 & 0.50 & 0.08 & 0.04 \\
4 & 0.30 & 0.01 & 0.04 & 0.04 & 0.56 & 0.83 & 0.02 & 0.05 \\
\hline
\end{tabular}

\section{DISCUSSION}

The results in the present investigation are supported by the observations that the mixing of domestic wastes favours the growth of characteristic plants even though heavy metal, nutrient, organic carbon contents, conductance and $\mathrm{pH}$ show higher values when domestic wastes mix with river Varuna [Agarwal et al (2000)] and 50\% concentration of sewage was ideal for better yield [Kannabiran and others (1998)]. In the present case also, it was observed that loamy soil treated with river water yields maximum biomass. Augusty et.al (2001) have shown that higher concentrations $(>50 \%)$ of rubber factory effluent reduce the percentage seed germination of Vigna radiatus $L$ while low concentrations favour seedling growth and length of shoot. Similar observations indicating reduced germination of seeds with increased effluent concentrations have been reported by Chidaunbalam Pillai (1996) with chemical effluents, Dutta (1997) with paper mill effluent, Pundir Suman (1995) with only Manganese on Zea Mays, Saravanan et.al (1996) with only Copper on green gram and Prasanna Kumar et.al (1997) with dairy effluent. Biney Charles A. (1991) finds that maize seeds are very sensitive to pollutants in wat its germination can be used as a tool to characterize natural and wastewater. De Wet et.al (1990) have shown that bioaccumulation of heavy metals by water fern-Azolla filiculoides Lam is not necessarily related to their natural concentration in the aquatic environment. This is confirmed by the fact that no accumulation of heavy metal was observed in the edible part though the river water contains dissolved heavy metals.

Guptha Anil (1997) has shown that presence of lead retards growth of both root and shoot in gram, lentil, and wheat and matra crops. Jeevan Rao and Shantaram (1995) have pointed out a potential pollution hazard of heavy metal contents in plants through their entry into the food chain when urban solid waste is used in fields. This is contradicted by (a) Singh et al (1993) with the observation on cabbage and spinach that $\mathrm{Pb}$ accumulates more in roots than shoots and (b) Lewander et al (1996) who have shown the accumulation of metals mainly in leaves of Potamogeton Pectinatus and Myriophyllum Verticillatum. This indicates that no accumulation og heavy metals in edible parts, which is found to be true even in the present case. Diamond et al (1994) have concluded that high effluent organic carbon was at least partly responsible for the occasional high levels of dissolved metals not causing toxicity to plant life. In the present analysis, also, the presence of heavy metals in river water and sediment did not cause toxicity to the growth of the plants due to high organic content in the water and sediment accompanied by slightly alkaline $\mathrm{pH}$ of both water and sediment and no heavy metal content was observed in the pods (the edible part). Mehra et.al (2000) has assigned the unhealthy growth of Eichhornia crassipes in the overbank of river Yamuna to the alkaline $\mathrm{pH}$ of growth medium, metal toxicity and high BOD. This is in contradiction to the 
present findings that alkaline $\mathrm{pH}$ leads to prevention of heavy metal toxicity.

Nagaraj et.al (1988) have found that number of tillers/clump and the plant heights of fodder grass were more in sewage-irrigated plots than well water. In the present study also, all the parameters studied such as plant height, biomass etc, were much more in the sewage affected river water. According to Pandit and Prasanna Kumar (1999) the growth of shoot and root of Jowar were reduced with respect to increase in metals concentration with total inhibition of germination at $100 \mathrm{ppm}$ concentration. Ponmurugan and Jayaseelan (1999) with some industrial effluents on Typha angustate, have reported similar observation. These workers have not indicated the $\mathrm{pH}$ of the water used for watering. $\mathrm{pH}$ plays a vital role in heavy metal toxicity studies as these are in the unavailable form only in slightly alkaline range and hence are of little significance. Sharma and Pant (1994) have shown that the $\mathrm{Cr}$ supply reduces the uptake of $\mathrm{Mn}, \mathrm{Zn}$ and $\mathrm{Cu}$ in Maize. Contradicting this Sinha et.al (1993) say that in Hydrilla vertucullata, $\mathrm{Mn}$ ameliorates $\mathrm{Cr}$ toxicity by inhibiting its uptake. Shanmugavel (1993) has concluded that sewage, paper and dye industry effluents delay the commencement of germination and reduced both percentage germination and germination value of green gram and maize seeds. This is supported by Srivastava et.al (1993) who has said that the Lettuce plant growth decreases in the sludge showing good fertilizer value when irrigated with sewage water instead of tap water, attributing this to the uptake beyond tolerable limits of $\mathrm{Cu}, \mathrm{Zn}$ and $\mathrm{Cr}$ present in toxic concentrations. These contradict the present findings, as the heavy metals were not found to pose any threat with no accumulation in the edible parts.

Conclusion: It is concluded that this river water and sediment might be used as a source of fertilizer for better growth of plants. Presence of heavy metals, though high in water and sediment does not enter the food chain and hence poses no threat.

Acknowledgements: The authors thank the management of P.E.S.Degree College, Bangalore and the Kuvempu University, Shankaraghatta for providing facilities for research and UGC for financial support. They are also grateful to Mrs. Hema Ahipathy for her help in collecting samples.

\section{REFERENCES}

Ahipathy, M V; Puttaiah, E T (2006) Ecological characteristics of Vrishabhavathy River in Bangalore(INDIA). Envi Geol 49(8): 1217-1222.
Aggarwal, T R; Singh, K N; Gupta, A K (2000). Impact of sewage containing domestic wastes and heavy metals on the chemistry of Varuna river water. Pollution Research 19(3): 491-494.

Augusthy, P O; Mani, A S (2001). Effect of factory effluent on seed germination and seedling growth of Vigna radiatus L. Journal of Environmental Research 22(2): 137-139.

Biney, C A (1991). A method for characterizing natural and wastewaters using plant seeds. Environmental Monitoring and Assessment 18(2): 123-28.

Chidaunbalam, P S; Pugazhendi, N; Lakshmanan, C; Shanmugasundaram, R (1996). Effect of chemical industry waste water on germination, growth and some biochemical parameters of Vigna radiata L. Wilcseck and Vigna mungo L. Heppter. Journal of Environmental Pollution 3(3\&4): 131-134.

De Wet, L P D; Schoonbee, H J; Pretorius, J; Bezuidenhout, L M (1990). Bioaccumulation of selected heavy metals by the water fern, Azolla filiculoides lam in a wetland ecosystem affected by sewage, mine and industrial pollution. Water SA 16(4): 281-86.

Diamond, J M; Hall, J C; Pattie, D M; Gruber, D (1994). Use of an integrated monitoring approach to site-specific effluent metal limits. Water Environment Research 66(5): 733-43.

Dutta, S K; Boissya, C L (1997). Effect of paper mill effluent on germinations of rice seed (Oryza sativa L. var. Masuri) and growth behaviour of its seedlings. Journal of Industrial Pollution Control 13(1): 41-47.

Gupta, A K R; Sharma, S (1997). Growth response of some crop-seedings to heavy metal (lead) treatment. Journal for Nature Conservation 9(1): 27-30.

Jeevan R K; Shantaram, M V (1995). Contents of heavy metals in crops treated with urban solid wastes. Journal of Environmental Biology 16(3): 225-232.

Kannabiran, B; Harilal, C C (1998). Studies on the effect of domestic sewage on the growth and yield of Vigna mungo (L) Hepper. Pollution Research 17(1): 33-37. 
Karmegam, N; Daniel, T (2000). Effect of biodigested slurry and vermicompost on the growth and yield of cowpea Vigna unguiculata (L.) Walp. Variety $\mathrm{Cl}$. Environment and Ecology 18(2): 367-370.

Lewander, M; Gregar, M; Kautsky, L (1996). Macrophytes as indicators of bioavailable $\mathrm{Cd}, \mathrm{Pb}$ and $\mathrm{Zn}$ flow in River Przemsza, Katowice Region. Applied Geochemistry 11(1-2): 169173.

Mehra, A; Farago, M E; Banerjee, D K (2000). A study of Eichhornia crassipes growing in the overbank and floodplain soils of the River Yamuna in Delhi, India. Environment Monitoring and Assessment 60(1): 24-25.

Nagaraja, D N; Krishnamurthy, K (1988). Raw sewage on the growth and yield of fodder grass (NB-21). Mysore Journal of Agricultural Science 22(3), 305-309.
Prasanna, K P G; Pandit, B R; Mahesh, K R (1997). Effect of dairy effluent on seed germination, seedling growth and pigment content of green gram (Phaseolusaureus L.) and black gram (Phaseolus mango L.). Advances in Plant Sciences 10(1): 129-136.

Pundir, S (1995). Retardation and acceleration of seed germination and seedling growth in the presence of heavy metal Mn. Advances in Plant Sciences 8(1) (Supp): 91-95.

Saravanan, S; Subramani, A; Lakshmanachary, A S (1996). Effect of copper sulphate on seed germination and early seedling growth of green gram (Vigna Radiata Linn. Wilczek). Ecology and Environment Conservation. 2(3\&4): 163164.

Shrivastava, G K; Singh, V P (1990). Effect of municipal waste water on yield and heavy metal contents of Abelmoschus esculentus (L). Moeunch Oikoassay 7(1\&2): 5-7. 